\title{
REPORT OF THE ARCHAEOLOGY WORKSHOP, SUNDAY 14 AUGUST 1994
}

\section{MARK VAN STRYDONCK}

\author{
Royal Institute for Cultural Heritage, Jubelpark 1, B-1040 Brussels, Belgium
}

The morning session of the Archaeology Workshop focused on the recolonization of Europe after the 18,000 BP glaciation. Five papers were presented. Two papers, one by Jacobi and Housley and the other by Woodman discussed bone-dating projects from the British Isles.

AMS dating of bones showed that, although many animal species occurred in England and Wales both before and after the Late Glacial Maximum, humans were not detected during the period $23,000-12,800 \mathrm{BP}$. Human activity was revealed by butchery marks on bones as well as formal bone and antler artifacts. The program of dating Ireland's fauna demonstrated a rich range of mammalian remains before the late Midlandian ice sheet retreat. Late Glacial fauna were similar to the British fauna but less abundant. It seems possible that the origin of some elements in the Postglacial fauna may be found for the Late Glacial.

Ruth Charles discussed AMS dating of bone from the Ardennes caves in Belgium. She also used carve-marked bones for her investigation. Different caves gave an average date of $c a .12,800 \mathrm{BP}$ for the earliest human recolonization.

Gamble and Housley presented new data from the Swabian caves, suggesting that Magdalenian reoccupation began ca. 13,300-13,200 BP. Expansion into the North European Plain and Southern Scandinavia occurs later as shown by the many Hamburgian sites, which postdate $13,000 \mathrm{BP}$.

A final morning paper presented by Evin, Bintz and Monjuvent (1994) discussed human settlements and the Würmian deglaciation in the French Alps. Geological samples seem to be inadequate to date the ice retreat. A review of radiocarbon dates from Paleolithic sites showed that no sites older than 15,000 BP could be found east of the Rhône, even at low altitude. The mountain region seems to have been occupied rather suddenly at $c a .13,500 \mathrm{BP}$, suggesting no direct connection between ice retreat and human settlement.

The subject of recolonization was discussed further in the first afternoon session, and can be summarized around one question: "Do our dates reflect what happened?" or, as Erle Nelson posed more astutely, "We are looking at the difference between evidence of lack and lack of evidence." All dates seem to focus on a period from 13,000 to 12,000 BP, but we do not know if deposition and preservation of artifacts are not subject to some natural selection criteria of which we are not aware. Almost all samples come from caves. A detailed understanding of formation processes is essential, particularly because a large proportion of each layer consists of naturally formed material. Also, it is well known that cave deposition is not a continuous process and that deposits are often highly perturbed. Further, we lack important information from open-air settlements. Some of these sites are difficult to recognize, especially if one has to search material stored in old museum collections.

We cannot measure the true duration or rate of recolonization until we know the shape of the calibration curve; the coral-based spline suggests major fluctuations during this period. Some participants argued that even a plateau of $c a .300 \mathrm{yr}$ in the curve would not really affect the observed phenomenon. 
The second afternoon session was reserved for general discussion. The first topic was calibration. Some archaeologists were uncomfortable that both 1986 and 1993 calibrations are in use and give different results.

Going from calibration to precision, not only in the calibration curve, but also in the dates laboratories produce, was only a small step. Attendees felt that accuracy is more important than precision, and that archaeological accuracy is often less than ${ }^{14} \mathrm{C}$ accuracy.

A second group of topics dealt with the relation between ${ }^{14} \mathrm{C}$ dates and the archaeological event one wishes to date. The sample reflects predepositional and postdepositional processes. Human activities, as natural processes, can lead to mixing of old and young material, even (in the case of cave deposits) below undisturbed layers of calcite. Following this is the question of synchronization of event and date. It was argued that archaeologists are too lenient about the source of their material and that rigorous questions about sample integrity are seldom asked.

Also discussed were the validity of stratigraphy as a marker for succession and the validity of styles in pottery and artifacts as chronological tools. One school of thought held that use of typologies for chronological information prevented understanding of processes in time. Another school agreed that typologies provide useful, if crude, chronological information. Differences of opinion remained unresolved. The use of these parameters seemed to depend strongly on the kind of problems one wants to solve and the particular situation of the site or the material being studied. A major question was inclusion or exclusion of "bad" dates in studies of large numbers of dates. Some thought this could be dealt with by discarding extreme dates on the basis of the typology of the entities dated, which then led to further discussion on the problem of "old" dates. By this, members meant dates measured 20 or more years ago. The question was asked if there should be a protocol for discarding old dates. Most were against this proposal as well as that of rejecting all dates measured $>20 \mathrm{yr}$ ago. The problem is that it is very difficult to devise a protocol that includes the archaeological as well as the chemical aspects, the counting and the statistical evaluation of the dates.

Quality assurance was the following topic of discussion. There seems to be "political" pressure to go to formal quality assurance, using international reference standards. This was confirmed by representatives from the UK and New Zealand. Governmental agencies will almost certainly implement quality assurance in contracts. Unfortunately, people from continental Europe or the USA did not react to this. Thus, we do not know how we stand and what impact this normalization will have. The point was made that quality assurance must be applied equally to the archaeological part of the dating process if we are to obtain accurate dates relevant to archaeological questions.

\section{REFERENCE}

Evin, J., Binz, P. and Monjuvent, G. 1994 Human settlements and the last deglaciation in the French Alps. Radiocarbon 36(3): 345-357. 